\title{
Frequency analysis of vehicle drive with cable traction
}

\author{
Serhii Raksha ${ }^{1}$, Oleksii Kuropiatnyk ${ }^{1, *}$, Pavlo Anofriev ${ }^{1}$, Dmytro Onopreychuk ${ }^{2}$, and Ihor \\ Kovalov $^{3}$ \\ ${ }^{1}$ Department of Applied Mechanics and Materials Science, Dnipropetrovsk National University of \\ Railway Transport named after Academician V. Lazaryan, Dnipro, Ukraine \\ ${ }^{2}$ Department of Construction, track and handling machines, Ukrainian State University of Railway \\ Transport, Feierbakh Square 7, 61050, Kharkiv, Ukraine \\ ${ }^{3}$ National Academy of the National Guard of Ukraine, Defenders of Ukraine Square 3, 61001, \\ Kharkiv, Ukraine
}

\begin{abstract}
The paper outlines the basic principles of constructing frequency diagrams and specifies the features of their application for the analysis of the dynamic state of the vehicle drive with cable traction on the example of a ropeway. To draw up the frequency diagrams, the ropeway traction circuit was presented as a dynamic system containing lumped masses and elements with distributed parameters. In this case, the moments of inertia of the rotating masses of the drive were brought to the traction pulley as a lumped mass. The traction cable segments are represented as elements with distributed parameters. The mathematical model describing the dynamic state of the drive consists of equations in the form of deformations of the traction cable segments using the positions of wave mechanics. Studies have shown that in most cases there is a fairly wide inter-resonance band, limited by the first and second eigenfrequencies. This phenomenon may be the basis for justifying the rational values of the rotational speed of the pulley as one of the kinematic parameters of the ropeway drive.
\end{abstract}

\section{Introduction}

A lot of means of industrial transport have a flexible traction unit, among them belt and chain conveyor; vertical and inclined mine lifts. These machines also include tools with cable traction: hanging conveyors, monorail cable cars and ropeways. They differ in industries and technology, but the common feature is the presence of a drum drive (for belt conveyors), with one sprocket or a few sprockets (for chain conveyors and some hanging conveyors), or with a traction pulley (for mine lifts and ropeways). Instability of its operation (in particular, during transient modes) can lead to emergency situations. Therefore, the studies of the dynamic state of the vehicle drive with cable traction in order to prevent processes that disrupt its normal operation are relevant.

One of the measures to prevent the instability of the drive and to avoid emergencies

\footnotetext{
* Corresponding author: kuropyatnick@gmail.com
} 
when using the means of industrial transport is to limit the speed of the traction unit and the associated working elements (scrapers for the chain conveyor, overhead conveyor car, cable car, etc.). However, this does not allow controlling the dynamic processes during the startup period, in particular, to prevent and limit resonant phenomena.

To analyze the eigenfrequency spectrum of a vehicle drive with a cable traction under which there are resonance phenomena, it is convenient to use frequency diagrams that reflect the eigenfrequency as a function of moving the working element. By imposing on such diagrams a tachogram of the drive, it is possible to conclude about the safety of the vehicle as a dynamic system, determining the number of hazardous resonant zones that the drive pass during acceleration and deceleration, as well as the frequency and (for certain additional calculations) amplitudes of oscillations in the resonant zones. In addition, the inverse problem solution allows the development of rational tachograms of the drive to prevent the occurrence of resonant phenomena.

Consider the features of the frequency analysis of the vehicle drive with cable traction on an example of a ropeway.

\section{Analysis of publications}

Some results of research on rational design and effective use of ropeway elements are given in [1-3]. However, most of them are due to calculations based on static formulas.

The work [4] is devoted to study on the dynamic load of ropeway elements. That paper considers the processes in traction ropes, but only transverse oscillations are taken into account; the effect of these processes on the dynamics of the drive is not taken into account.

The importance of applying an integrated approach to evaluate the load of ropeway elements is confirmed by foreign experience [5-10]. However, the recommendations based on the analysis of the eigenfrequency spectrum of the drive have not been detected.

The formation of basic approaches to the construction and analysis of the eigenfrequency spectrum of the drive by frequency diagrams was begun in the works [11, 12]. However, the materials contained therein don't apply to ring-type ropeways.

\section{Purpose of work}

The purpose of this work is to develop the basic principles of constructing frequency diagrams and to formulate approaches to the application of such diagrams during the analysis of the dynamic state of vehicle drives with cable traction on the example of ropeway.

\section{Theoretical studies}

To compile frequency diagrams of the drive, we consider the traction circuit of cableway as a dynamic system, which includes a drive, a traction cable with a tensioner and cars (Fig. 1). In this case, we replace the components of the drive by three lumped masses - the rotor of the electric motor, the gear unit and the pulley. These masses are interconnected by elastic weightless connections. The cars and the tensioner are interconnected by segments of a traction cable as elements with distributed parameters.

In this problem statement, a mathematical model describing the dynamic state of a system consists of differential equations in ordinary derivatives and partial differential equations. Solution the system of this equation in order to obtain the frequency function is complicated, so within the scope of this work we perform the replacement the schema shown in Fig. 1 by simplified schema (Fig. 2). The peculiarity of this approach is to bring 
the masses of the drive to the pulley, which allows us to restrict the use of differential equations in partial derivatives during the compilation of a mathematical model.

In order to formulate the description of the mathematical model, we introduce the following concepts:

- number of cars in one direction of travel $(n)$ - the number of cars that are simultaneously located on the area between the pulley and the tensioner as lumped masses (constructively between the drive and tension stations) and move in one direction; thus the total number of cars is $2 n$;

- lower car - a car moving from the lower station to the upper;

- upper car - a car moving from the upper station to the lower one;

- lower direction of movement - the section of the route, along which the lower cars move;

- upper direction of movement - the section of the route, along which the upper cars move.

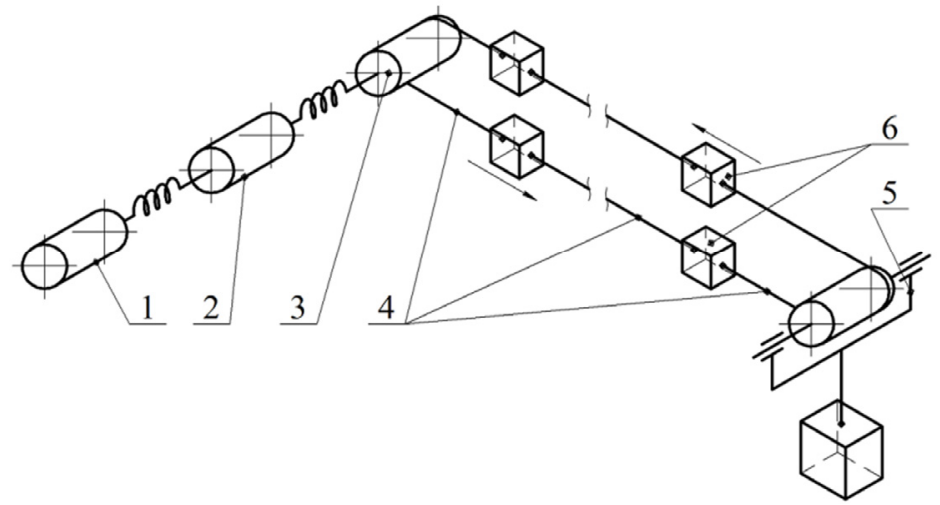

Fig. 1. Ropeway traction circuit: 1 - the rotor of the electric motor; 2 - mass of the gear unit; 3 - pulley; 4 - the traction cable segments; 5 - tensioner; 6 - cars

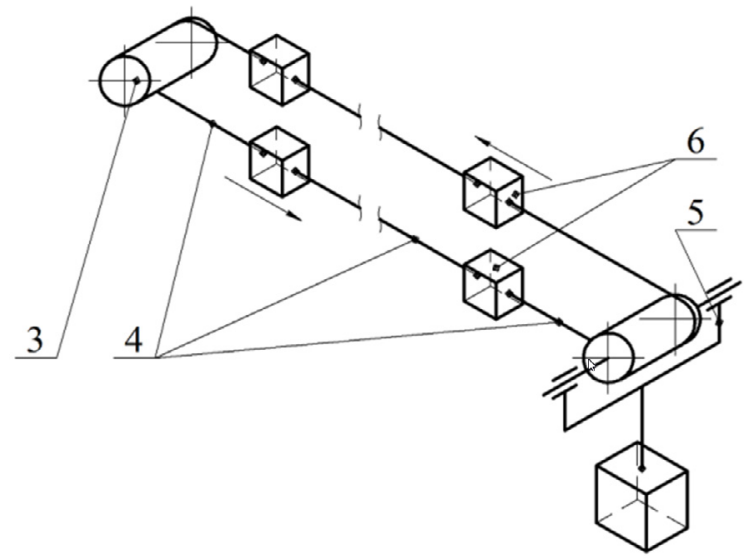

Fig. 2. Simplified model of the ropeway traction circuit (designation according to Fig. 1)

In absolute terms these statements are valid for ropeways, whose profile changes monotonically; that is the difference in elevation between two adjacent reference points in the upper direction of motion is always positive, and in the lower direction of motion it is negative. In the case of changes in the profile curvature, these concepts can be used for each span individually, and then combine into a common simulation system. 
For the convenience of compiling a mathematical model we use a substitution scheme in which all masses make translational movement [13] (Fig. 3, a). In this case, the feature of such a scheme for the traction circuit of ropeway is its closeness, which determines a certain specificity of the formation of equations. It is reflected in the application of additional forces that characterize the mutual influence of finite masses (Fig. 3, b).

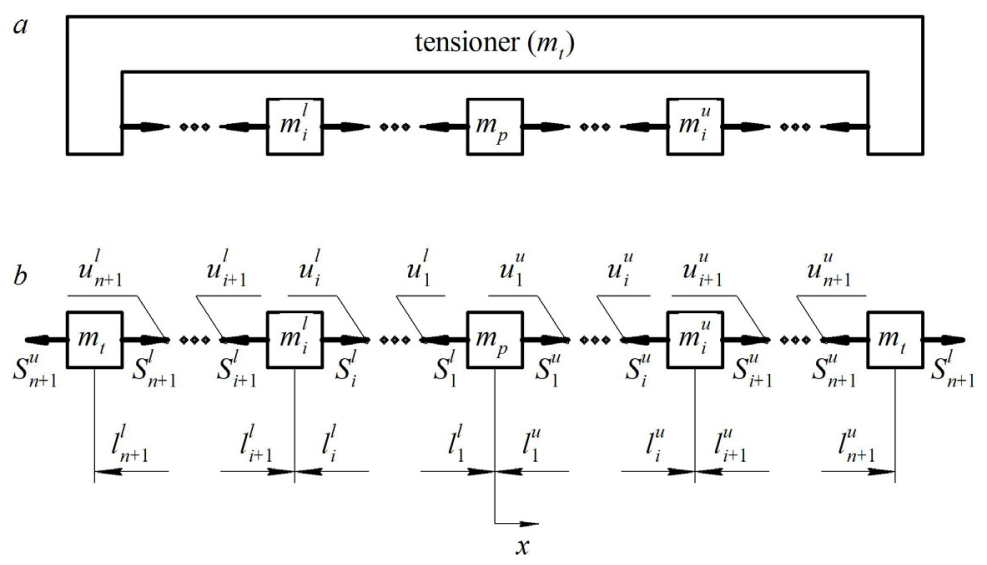

Fig. 3. Substitution scheme: $a$ - general view; $b$ - with additional force applied

In the substitution scheme we use the following notation:

$m_{p}$ - mass of the pulley (equivalent weight of all elements of the drive);

$m_{t}$ - mass (equivalent to the working force) of the tensioner;

$m_{i}^{l}$ - mass of the $i$-th lower car $(i=\overline{1, n})$;

$m_{i}^{u}$ - mass of the $i$-th upper $\operatorname{car}(i=\overline{1, n})$;

$u_{1}^{l}$ - elastic deformation of the traction cable segment between the pulley and the first car in the lower direction of movement;

$u_{i}^{l}$ - elastic deformation of the traction cable segment between (n-1)-th and $i$-th cars in the lower direction of movement $(i=\overline{2, n})$;

$u_{n+1}^{l}$ - elastic deformation of the traction cable segment between the $i$-th car in the lower direction of movement and the tensioner;

$u_{1}^{u}$ - elastic deformation of the traction cable segment between the pulley and the first car in the upper direction of movement;

$u_{i}^{u}$ - elastic deformation of the traction cable segment between $(n-1)$-th and $i$-th cars in the upper direction of movement $(i=\overline{2, n})$;

$u_{n+1}^{u}$ - elastic deformation of the traction cable segment between the $i$-th car in the upper direction of movement and the tensioner;

$S_{1}^{l}, S_{i}^{l}, S_{n+1}^{l}, S_{1}^{u}, S_{i}^{u}, S_{n+1}^{u}$ - elastic forces that correspond to deformations $u_{1}^{l}, u_{i}^{l}$, $u_{n+1}^{l}, u_{1}^{u}, u_{i}^{u}, u_{n+1}^{u}$

$$
S_{i}^{l}=E F \frac{\partial u_{i}^{l}}{\partial x} ; \quad S_{i}^{u}=E F \frac{\partial u_{i}^{u}}{\partial x}, \quad i=\overline{1, n+1},
$$

$E, F$ - the elastic modulus and the area of the traction cable cross-section. 
According to the indicated symbols, the mathematical model describing the dynamic state of the drive consists of equations in the form of deformations of the traction cable segments using the positions of wave mechanics [13]:

$$
\left\{\begin{array}{l}
\frac{\partial^{2}}{\partial t^{2}} U^{l}=a^{2} \frac{\partial^{2}}{\partial x^{2}} U^{l} \\
\frac{\partial^{2}}{\partial t^{2}} U^{u}=a^{2} \frac{\partial^{2}}{\partial x^{2}} U^{u}
\end{array}\right.
$$

$U^{l}=\left[u_{i}^{l}\right]$ - vector of deformations of the traction cable segments on the lower direction of the car movement $(i=\overline{1, n+1}) ; U^{u}=\left[u_{i}^{u}\right]$ - vector of deformations of the traction cable segments on the upper direction of the car movement $(i=\overline{1, n+1}) ; a$ - the propagation velocity of the elastic wave in the longitudinal direction of oscillation.

In order to simplify the recording, we introduce the following notation:

$x_{i}^{l}=-\sum_{j=1}^{i} l_{j}^{l}$ - coordinate of the $i$-th car in the lower direction of movement;

$x_{i}^{u}=\sum_{j=1}^{i} l_{j}^{u}$ - coordinate of the $i$-th car in the upper direction of movement;

$\alpha_{i}^{l}=q l / m_{i}^{l}-$ mass coefficient of the $i$-th car in the lower direction of movement;

$\alpha_{i}^{u}=q l / m_{i}^{u}$ - mass coefficient of the $i$-th car in the upper direction of movement;

$\alpha_{p}=q l / m_{p}-$ mass coefficient of the pulley;

$\alpha_{t}=q l / m_{t}-$ mass coefficient of the traction cable tensioner;

$\lambda=k l-$ the actual number of the frequency function ( $k$ is a certain constant used in the transformation of the system of equations [13]);

$A, B$ (with any indexes) - amplitude coefficients.

Also consider the following parameters of the traction cable: $E$ - elastic modulus; $F$ - cross-section area; $q$ - linear mass; $l$ - the length of the traction cable segments between the pulley and the tensioner as lumped masses in one direction of movement.

For equations in the form of the first expression of the system (2), which describe the movement of the $i$-th car in the lower direction, the boundary conditions have the form $(i=\overline{1, n})$ :

$$
\left.m_{i}^{l} \frac{\partial^{2} u_{i}^{l}}{\partial t^{2}}\right|_{x=x_{i}^{l}}=\left.E F\left(\frac{\partial u_{i}^{l}}{\partial x}-\frac{\partial u_{i+1}^{l}}{\partial x}\right)\right|_{x=x_{i}^{l}} ; \quad u_{i}^{l}\left(x_{i}^{l}, t\right)=u_{i+1}^{l}\left(x_{i}^{l}, t\right) .
$$

For equations in the form of the second expression of the system (2), which describe the movement of the $i$-th carriage in the upper direction, the boundary conditions have the form $(i=\overline{1, n})$ :

$$
\left.m_{i}^{u} \frac{\partial^{2} u_{i+1}^{u}}{\partial t^{2}}\right|_{x=x_{i}^{u}}=\left.E F\left(\frac{\partial u_{i+1}^{u}}{\partial x}-\frac{\partial u_{i}^{u}}{\partial x}\right)\right|_{x=x_{i}^{u}} ; \quad u_{i}^{u}\left(x_{i}^{u}, t\right)=u_{i+1}^{u}\left(x_{i}^{u}, t\right) .
$$

To combine the equations (3) and (4) with the system and to ensure the closeness of the traction circuit, we use the conjugation conditions at the traction cable segments near the pulley and the tensioner. We make such expressions in the form of boundary conditions to 
differential equations which describe the movement of the pulley and the tensioner as lumped masses $m_{p}$ and $m_{t}$ respectively.

According to the above notation, the boundary conditions for the equation describing the movement of the pulley have the form:

$$
\left.m_{p} \frac{\partial^{2} u_{1}^{u}}{\partial t^{2}}\right|_{x=0}=\left.E F\left(\frac{\partial u_{1}^{u}}{\partial x}-\frac{\partial u_{1}^{l}}{\partial x}\right)\right|_{x=0} ; \quad u_{1}^{l}(0, t)=u_{1}^{u}(0, t),
$$

$x=x_{1}^{u}=x_{1}^{l}=0-$ pulley coordinate.

The boundary conditions for the equation describing the motion of the tensioner are as follows:

$$
\left.m_{t} \frac{\partial^{2} u_{n+1}^{l}}{\partial t^{2}}\right|_{x_{n+1}^{l}}=E F\left(\left.\frac{\partial u_{n+1}^{l}}{\partial x}\right|_{x_{n+1}^{l}}-\left.\frac{\partial u_{n+1}^{u}}{\partial x}\right|_{x_{n+1}^{u}}\right) ; u_{n+1}^{u}\left(x_{n+1}^{u}, t\right)=-u_{n+1}^{l}\left(x_{n+1}^{l}, t\right) .
$$

After separating the variables in equations (3)-(6), we combine the results obtained with the system (7).

$$
\begin{aligned}
& \begin{aligned}
\int\left[\sin \left(\lambda \frac{x_{i}^{l}}{l}\right)-\frac{\lambda}{\alpha_{i}^{l}} \cos \left(\lambda \frac{x_{i}^{l}}{l}\right)\right] A_{i}^{l}- & {\left[\frac{\lambda}{\alpha_{i}^{l}} \sin \left(\lambda \frac{x_{i}^{l}}{l}\right)+\cos \left(\lambda \frac{x_{i}^{l}}{l}\right)\right] B_{i}^{l}-} \\
& -\sin \left(\lambda \frac{x_{i}^{l}}{l}\right) A_{i+1}^{l}+\cos \left(\lambda \frac{x_{i}^{l}}{l}\right) B_{i+1}^{l}=0 ;
\end{aligned} \\
& \cos \left(\lambda \frac{x_{i}^{l}}{l}\right) A_{i}^{l}+\sin \left(\lambda \frac{x_{i}^{l}}{l}\right) B_{i}^{l}-\cos \left(\lambda \frac{x_{i}^{l}}{l}\right) A_{i+1}^{l}-\sin \left(\lambda \frac{x_{i}^{l}}{l}\right) B_{i+1}^{l}=0 \\
& \sin \left(\lambda \frac{x_{i}^{u}}{l}\right) A_{i}^{u}-\cos \left(\lambda \frac{x_{i}^{u}}{l}\right) B_{i}^{u}-\left[\sin \left(\lambda \frac{x_{i}^{u}}{l}\right)-\frac{\lambda}{\alpha_{i}^{u}} \cos \left(\lambda \frac{x_{i}^{u}}{l}\right)\right] A_{i+1}^{u}+ \\
& +\left[\frac{\lambda}{\alpha_{i}^{u}} \sin \left(\lambda \frac{x_{i}^{u}}{l}\right)+\cos \left(\lambda \frac{x_{i}^{u}}{l}\right)\right] B_{i+1}^{u}=0 \\
& \cos \left(\lambda \frac{x_{i}^{u}}{l}\right) A_{i}^{u}+\sin \left(\lambda \frac{x_{i}^{u}}{l}\right) B_{i}^{u}-\cos \left(\lambda \frac{x_{i}^{u}}{l}\right) A_{i+1}^{u}-\sin \left(\lambda \frac{x_{i}^{u}}{l}\right) B_{i+1}^{u}=0 ; \\
& \begin{array}{l}
\frac{\lambda}{\alpha_{p}} A_{1}^{u}+B_{1}^{u}-B_{1}^{l}=0 ; \\
A_{1}^{u}-A_{1}^{l}=0 ;
\end{array} \\
& \sin \lambda \cdot A_{n+1}^{u}-\cos \lambda \cdot B_{n+1}^{u}+\left[\sin \lambda+\frac{\lambda}{\alpha_{t}} \cos \lambda\right] A_{n+1}^{l}-\left[\frac{\lambda}{\alpha_{t}} \sin \lambda-\cos \lambda\right] B_{n+1}^{l}=0 ; \\
& \cos \lambda \cdot A_{n+1}^{u}+\sin \lambda \cdot B_{n+1}^{u}+\cos \lambda \cdot A_{i+1}^{l}-\sin \lambda \cdot B_{n+1}^{l}=0 .
\end{aligned}
$$

The frequency function is established as a determinant of the matrix of the coefficients of the system (7). The zero arguments of this function (such that the function acquires a null value) are the eigenvalues $\lambda$, by which we determine the eigenfrequencies of the ropeway traction circuit as a dynamic system: 


$$
\omega=\frac{\lambda}{l} \sqrt{\frac{E F}{q}} .
$$

Taking into account that the ropeway traction circuit is modeled as a system of lumped masses (pulley, cars and tensioner) connected by elastic elements with distributed parameters (traction rope segments), the frequency function has a set of zero arguments, and hence the drive as the dynamic system has a set of eigenfrequencies $\omega$, which are determined by their eigenvalues $\lambda$.

We note that the frequency diagram shows the change of the eigenfrequencies of the drive during the movement of cars between stations. Examples of frequency diagram under the condition of the same loading of cars are shown in Fig. 4 (shows the first three eigenfrequencies). Compared to the second and third, the first frequency is relatively constant, since it is largely determined by the pulley mass. The coordinates of cars have a significant influence on the second and third eigenfrequencies, which explains the substantial nonlinearity of the corresponding curves. For a ring-type ropeway, there is a certain cyclic change in the third frequency, with the number of cycles corresponding to the number of cars in one direction of movement.
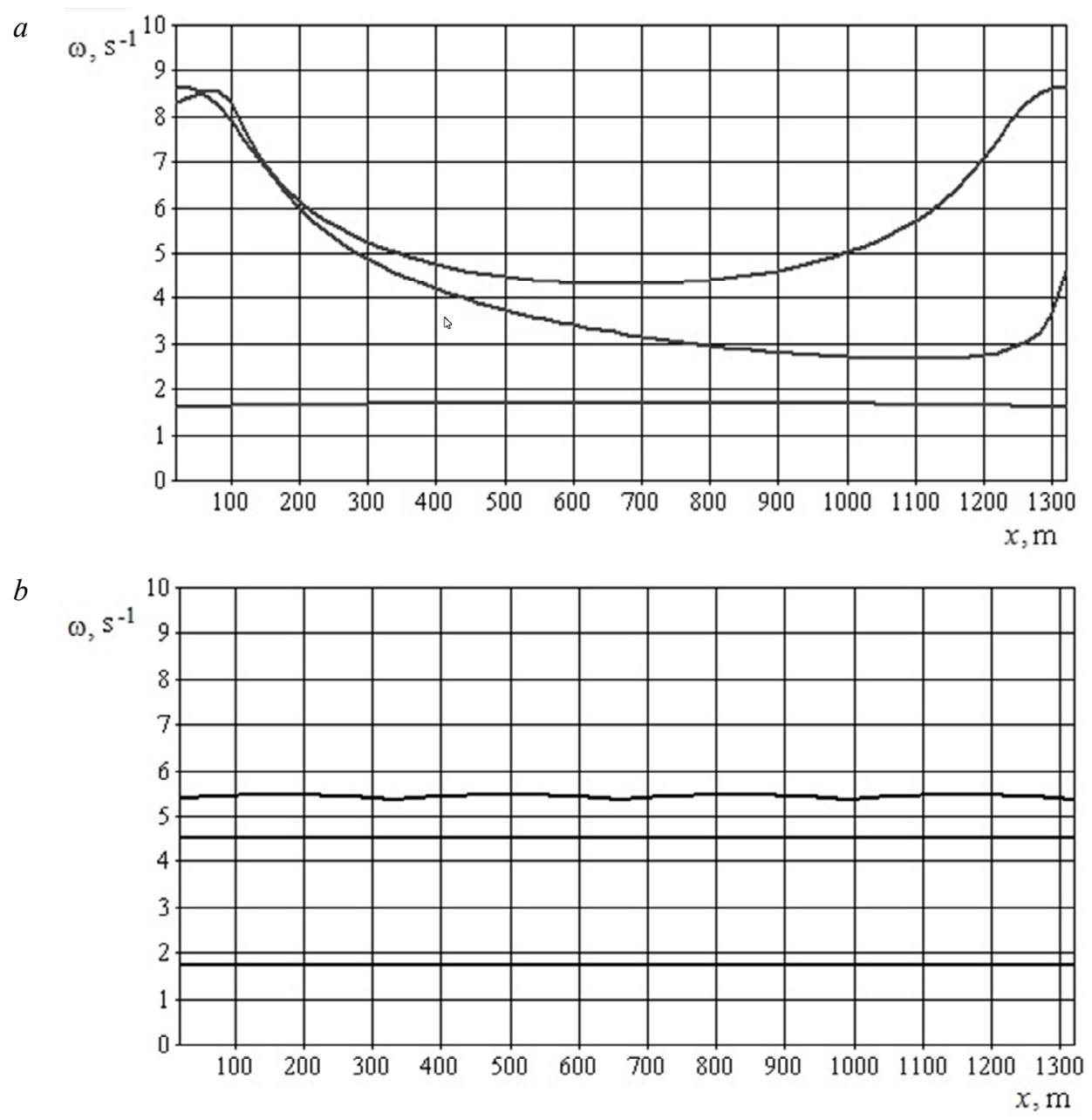

Fig. 4. Frequency diagrams of the ropeway drive: a - pendulum-type ropeway; $b$ - ring-type ropeway 


\section{Conclusions}

In most cases, there is a fairly wide interresonance band, limited by the first and second eigenfrequencies. This phenomenon may be the basis for justifying the rational values of the rotational speed of the pulley as one of the kinematic parameters of the ropeway drive. The results obtained are valid for all ropeways (both pendulum-type and ring-type) by the nature of the frequency diagram; however, the frequency values may vary, depending on the ropeway technical characteristics. For other vehicles with cable traction: for belt and chain conveyors, the degree of nonlinearity of frequency diagrams depends on the uniform distribution of cargo on the carrying surface; for hanging conveyors, the nature of the frequency diagrams is similar to that for a ring-type ropeways; frequency diagrams for mine lifts drives are similar to the pendulum-type ropeway diagrams but they are characterized by greater symmetry of the curves of the second and third eigenfrequencies for vertical lifting.

\section{References}

1. M. Berkman. Suspended cable cars (Mechanical Engineering, Moscow, 1984)

2. S. Raksha, O. Kuropiatnyk, A. Kurka. Science and Transport Progress. Bulletin of Dnipropetrovsk National University of Railway Transport 1 (49), 125-131 (2014)

3. Yu. Goriachev, O. Kuropiatnyk, M. Izmailov. Science and Transport Progress. Bulletin of Dnipropetrovsk National University of Railway Transport 3 (51), 109-116 (2014)

4. D. Pataraia. Calculation and design of cable systems on the example of ropeways (Mezniereba, Tbilisi, 1991)

5. J. Nejez. ISR 6, 47 (2011)

6. G. Kopanakis. ISR 6, 48-50 (2011)

7. G. Kopanakis. ISR 1, 46-49 (2012)

8. G. Kopanakis. ISR 3, 63-66 (2012)

9. J. Kowal. AME vol. LV 4, 357-368 (2008)

10. M. Knawa. PAMM vol. 8 1, 10297-10298 (2008)

11. S. Raksha, Yu. Goriachev, O. Kuropiatnyk. Construction, materials science, mechanical engineering 66, 249-256 (2012)

12. S. Raksha, Yu. Goriachev, O. Kuropiatnyk. Herald of the DSEA 1 (30), 110-116 (2013)

13. A. Stepanov. Dynamics of Machines (UBRAS, Ekaterinburg, 1999) 